\title{
Gross and histopathological pulmonary findings in a COVID-19 associated death during self-isolation
}

\author{
Christine Suess $^{1} \cdot$ Roland Hausmann ${ }^{1}$ \\ Received: 23 April 2020 / Accepted: 19 May 2020 / Published online: 5 June 2020 \\ (C) Springer-Verlag GmbH Germany, part of Springer Nature 2020
}

\begin{abstract}
Forensic investigations generally contain extensive morphological examinations to accurately diagnose the cause of death. Thus, the appearance of a new disease often creates emerging challenges in morphological examinations due to the lack of available data from autopsy- or biopsy-based research. Since late December 2019, an outbreak of a novel seventh coronavirus disease has been reported in China caused by "severe acute respiratory syndrome coronavirus 2" (SARS-CoV-2). On March 11, 2020, the new clinical condition COVID-19 (Corona-Virus-Disease-19) was declared a pandemic by the World Health Organization (WHO). Patients with COVID-19 mainly have a mild disease course, but severe disease onset might result in death due to proceeded lung injury with massive alveolar damage and progressive respiratory failure. However, the detailed mechanisms that cause organ injury still remain unclear. We investigated the morphological findings of a COVID-19 patient who died during self-isolation. Pathologic examination revealed massive bilateral alveolar damage, indicating early-phase "acute respiratory distress syndrome" (ARDS). This case emphasizes the possibility of a rapid severe disease onset in previously mild clinical condition and highlights the necessity of a complete autopsy to gain a better understanding of the pathophysiological changes in SARS-CoV-2 infections.
\end{abstract}

Keywords Forensic autopsy $\cdot$ Coronavirus · COVID-19 · Diffuse alveolar damage

\section{Introduction}

The appearance of a new disease generally creates emerging challenges in morphological examinations due to the lack of available data from autopsy- or biopsy-based research. Since late December 2019, an outbreak of a novel seventh coronavirus named "severe acute respiratory syndrome coronavirus 2" (SARS-CoV-2) has been reported in China. On March 11, 2020, COVID-19 was declared a pandemic by the WHO. As of April 8, 2020, approximately 1.44 million cases of COVID19 have been noted in 209 countries, resulting in approximately 82,900 deaths $[1,2]$. Before, six enveloped single-stranded RNA coronaviruses had been known causing pulmonary, hepatic, neurological, and intestinal disease in humans. The zoonotic "severe acute respiratory syndrome coronavirus" (SARS-CoV)

This article is part of the Topical Collection on COVID-19

Roland Hausmann

roland.hausmann@kssg.ch

1 Cantonal Hospital St. Gallen, Institute of Legal Medicine, Rorschacherstrasse 95, CH-9007 St. Gallen, Switzerland and the zoonotic "Middle East respiratory syndrome coronavirus" (MERS-CoV) can progress to a fatal course whereas the remaining four viruses usually cause mild cold symptoms [3]. Similar to SARS-CoV and MERS-CoV, SARS-CoV-2 attaches to the angiotensin-converting enzyme 2 (ACE 2) receptor which is usually present on the surface of alveolar cells of the lung epithelium, causing respiratory symptoms as the commonest presentation of COVID-19 [2]. Most patients initially present with a mild form of illness suffering from fever with or without respiratory symptoms [2]. Risk factors for severe illness or poor outcome tend to be old age, male gender, and many comorbidities, such as cardiovascular disease, hypertension, diabetes mellitus, cancer, immunosupression, and chronic respiratory failure $[4,5]$. An increased risk of death had been reported in men over the age of 60 and the case-fatality rate seemed to be elevated in patients with underlying diseases [2]. It is assumed that severe disease progression might result in death due to proceeded pulmonary injury with massive alveolar damage and progressive failure [2]. However, the detailed pathophysiological mechanisms that cause lung injury in SARS-CoV-2 infections still remain unclear. We investigated the morphological findings of a patient who died during self-isolation from severe infection with SARS-CoV-2, providing the opportunity for 
studying the pathology of COVID-19 in the complete forensic autopsy of a case without previous medical treatment.

\section{Case history}

A 59-year-old man with a medical history of hypertension (for the past 20 years) and type II diabetes presented to the family doctor for a routine examination where tachycardia (125 bpm), fever $\left(38.4^{\circ} \mathrm{C}\right)$, and a dry cough had been noticed. He did not specify other respiratory symptoms. A blood test revealed a decreased lymphocyte count. Due to the pandemic, a pharyngeal swab test was conducted. He received the diagnosis 'COVID 19' because of a positive test result for the novel coronavirus (SARS-CoV-2) and, therefore, he was transferred to self-isolation as he did not show severe respiratory symptoms. He received pain medication and a cough suppressant as a reliever. Five days later, he was found dead at home during a police inspection. He had been last seen alive just 24 hours prior to his discovery by the police without severe respiratory symptoms. Investigations at the scene showed no signs of external violence. To determine the cause of death, a complete forensic autopsy was performed.

\section{Morphological examinations}

The deceased man was $169 \mathrm{~cm}$ in height, weighed $74 \mathrm{~kg}$, and had a body mass index of $25.91 \mathrm{~kg} / \mathrm{m}^{2}$. The autopsy revealed no signs of external violence. The post-mortem computed tomography scan (CT scan) showed marked bilateral pulmonary ground-glass opacities and consolidations as well as small pleural and pericardial effusions (Fig. 1a). Both the left (weight $750 \mathrm{~g}$, normal 112-675 g) and the right lungs (weight $860 \mathrm{~g}$, normal 155-720 g) were enlarged [6] (Fig. 1b). Accumulation of serous fluids in both pleural cavities $(50 \mathrm{ml}$ right, $30 \mathrm{ml}$ left) and pericardial cavity $(30 \mathrm{ml})$ was noticed. Both lungs showed hemorrhage on the pleural surface without signs of pleurisy (Fig. 1b). Gross section of the lung revealed pulmonary edema and a diffusely firm and rubbery parenchyma with no palpable mass (Fig. 1c). All the lobes were affected equally. The bronchi were filled with fluid without
Fig. 1 a Representative image of post-mortem chest CT scan revealing bilaterally diffuse groundglass opacities and consolidations. b Lung, gross (inset: hemorrhage on the pleural surface). c Gross cross section of the right lung. d Gross cross section of the right lower lobe with fluid-filled Bronchi
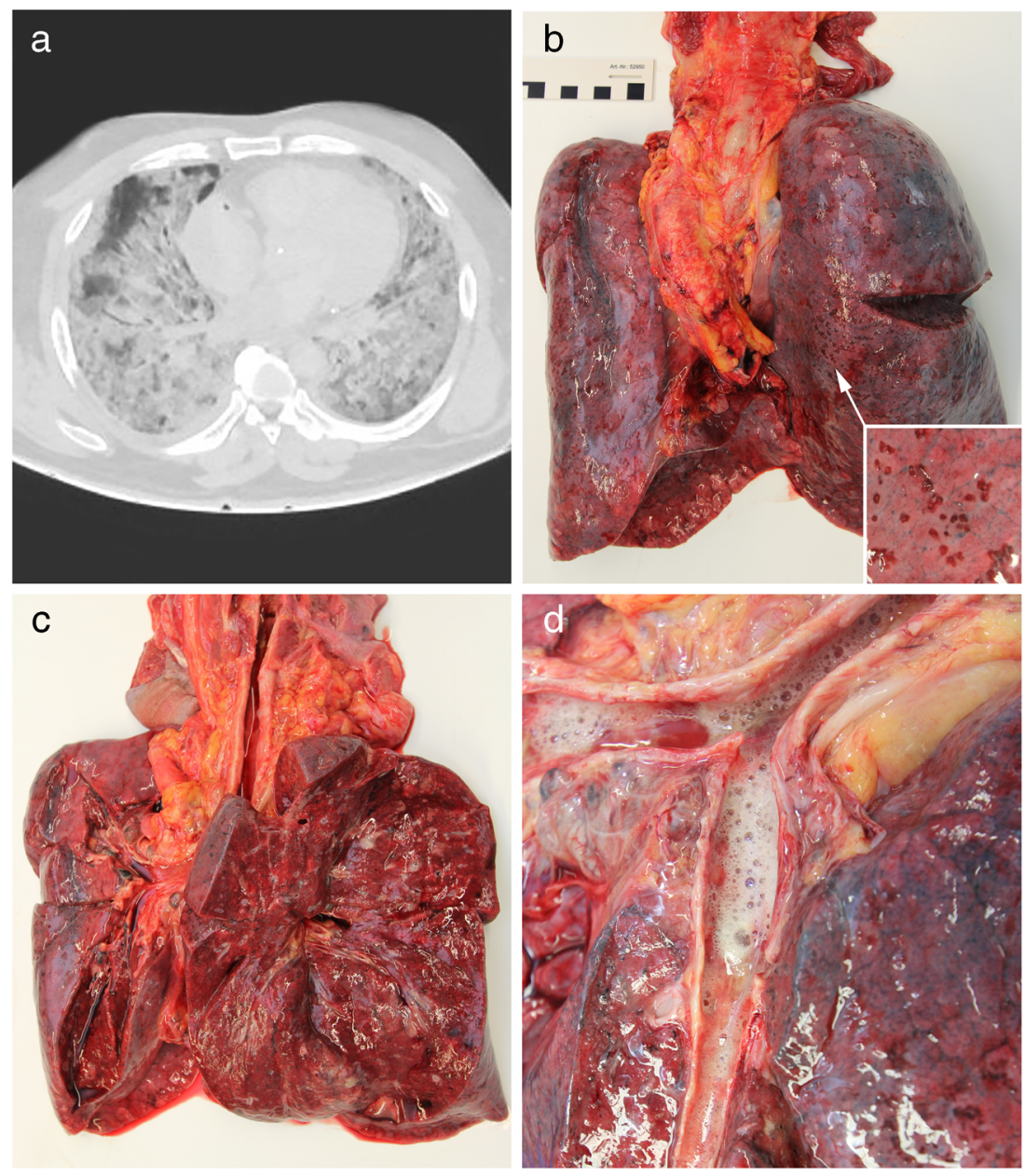
prominent mucus plugging and the vasculature was patent (Fig. 1d). The parenchyma had areas ranging from pale pink to dark red in color with scattered ill-defined hemorrhagic areas. The hilar lymph nodes were slightly enlarged with a white to black cut surface. Despite the long-term hypertension and type II diabetes, no significant changes could be detected in other organs. The brain weighed $1270 \mathrm{~g}$ and demonstrated no macroscopic lesion. To rule out other viral diseases, a postmortem swab test of lung tissue was performed that was negative for a great number of other virus causing respiratory disease (respiratory syncytial virus $\mathrm{A}$ and $\mathrm{B}$, rhinovirus $\mathrm{A}$, $\mathrm{B}$, and $\mathrm{C}$, influenza virus $\mathrm{A}$ and $\mathrm{B}$, human metapneumovirus, parainfluenza viruses $1-4$, adenovirus, enterovirus, bocavirus $1-4$, and coronavirus 229E, NL63, and OC43).

Multiple sections were taken for further microscopic examination. They were analyzed with routine hematoxylin and eosin (H\&E) staining and to some extent with periodic acidSchiff stain (PAS) to highlight hyaline basement membrane material and immunohistochemical staining for thyroid transcription factor-1 (TTF1) and clusters of differentiation 68 (CD68) to distinguish between pneumocytes (nuclear expression of TTF1) and macrophages (dot-like cytoplasmic or finely granular positivity for CD68). Routine staining of lung tissue revealed a predominance of pulmonary congestion and early stage diffuse alveolar damage with marked hyaline membrane formation, proteinaceous exudates, alveolar hemorrhage, and intra-alveolar fibrin deposition (Fig. 2a, b). In addition, there was a patchy distribution of intra-alveolar foamy macrophages filling some airspace throughout all lobes, proved by immunhistochemical staining with CD68 (Fig. 3a, b). The immunhistochemical staining with TTF1 confirmed patchy and severe type II pneumocyte hyperplasia characterized by atypically enlarged pneumocytes with large nuclei, amphophilic granular cytoplasm, and prominent nucleoli showing viral cytopathic-like changes and many mitotic figures (Fig. 2c, d). Obvious intranuclear or intracytoplasmic viral inclusions could not be detected. The bronchi revealed similar reactive changes of the epithelium with enlarged nuclei and increased mitotic figures (Fig. 3c). An increased number of intravascular megakaryocytes and a patchy mild increase of interstitial lymphocytic cells were noted. In addition, there were few areas of bronchial metaplasia with surrounding fibrosis of the interstitium. In the lower lobes, a focal neutrophilic infiltration was noted in some airspaces and the bronchial wall. There were no areas of organizing pneumonia with fibroblastic proliferations or Masson Bodies, and no architectural remodeling. No collections of granulomas were noted. In addition, hyaline microthrombi in pulmonary capillaries and some fresh thrombi in pulmonary arteries could be detected. The biopsy of the hilar lymph nodes revealed many noncaseous epithelioid cell granulomas as a nodal effacement of a previously unknown sarcoidosis. The liver specimen showed moderate macro- and microvesicular steatosis as well as some single necrotic hepatocytes around the central veins without portal or lobular inflammation. The biopsy samples taken from the heart showed patchy non-specific pericardial infiltration with aggregates of inflammatory cells, including lymphocytes and plasma cells (Fig. 3d). Neither neutrophils nor granulomas could be detected in the heart. There was no inflammatory infiltrate or substantial damage in the myocardium. Since the autopsy findings correlated with his medical history and the investigations at the scene, his cause of death was certified as ARDS due to severe diffuse alveolar damage as a result of severe infection with SARS CoV-2.
Fig. 2 Histologic changes in lung parenchyma (H\&E and TTF-1). a Low power demonstrating the predominance of acute diffuse alveolar damage. b Intermediate power demonstrating edema, hemorrhage, and fibrin deposition. c High power demonstrates atypical enlarged intra-alveolar cells characterised by large nuclei with increased mitotic figures (arrow). d Immunhistochemical staining with TTF-1 confirmed the atypical enlarged cells with type II pneumocytes
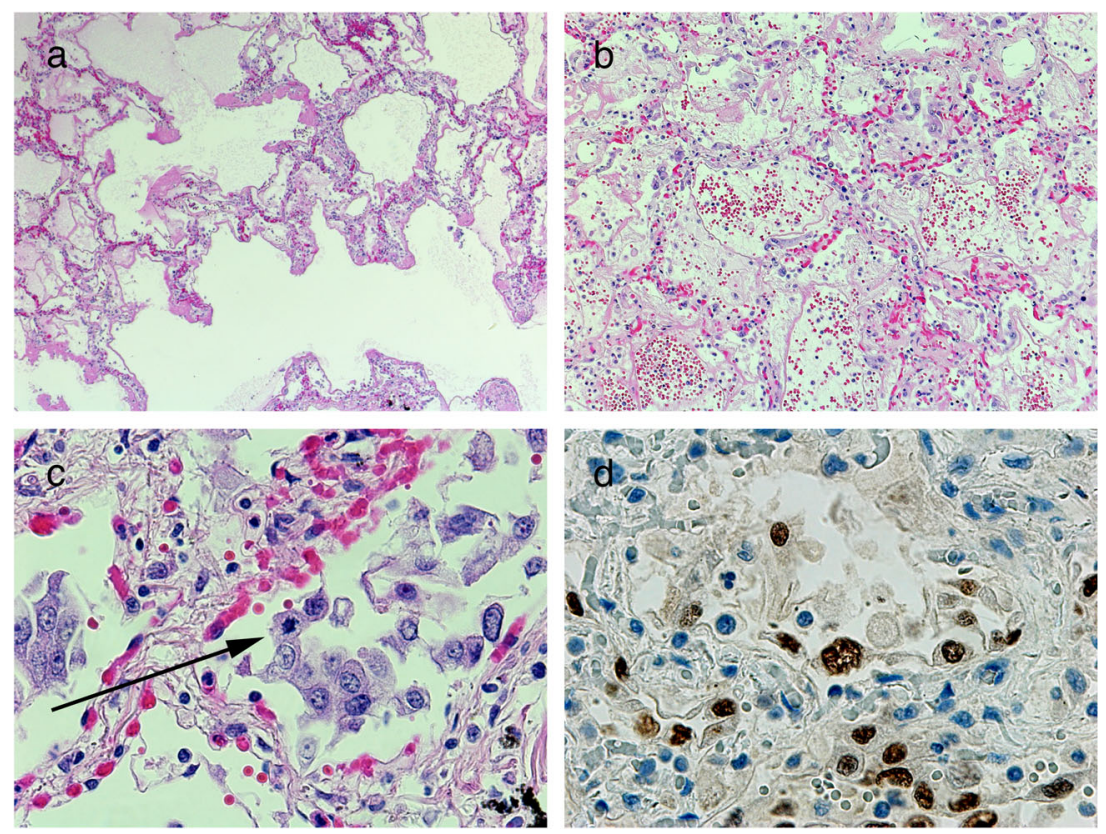


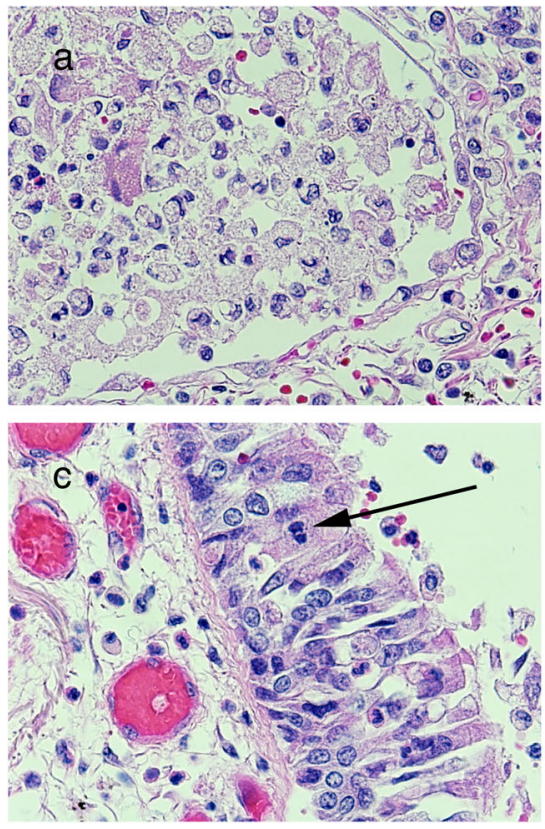

Fig. 3 Histologic changes in lung and heart parenchyma (H\&E and CD68). a High power demonstrating collections of intra-aveolar foamy macrophages. b Immunhistochemical staining with CD68 highlighted the abundance of macrophages in lung tissue. $\mathbf{c}$ High power demonstrating reactive changes of the bronchial epithelium with enlarged nuclei and

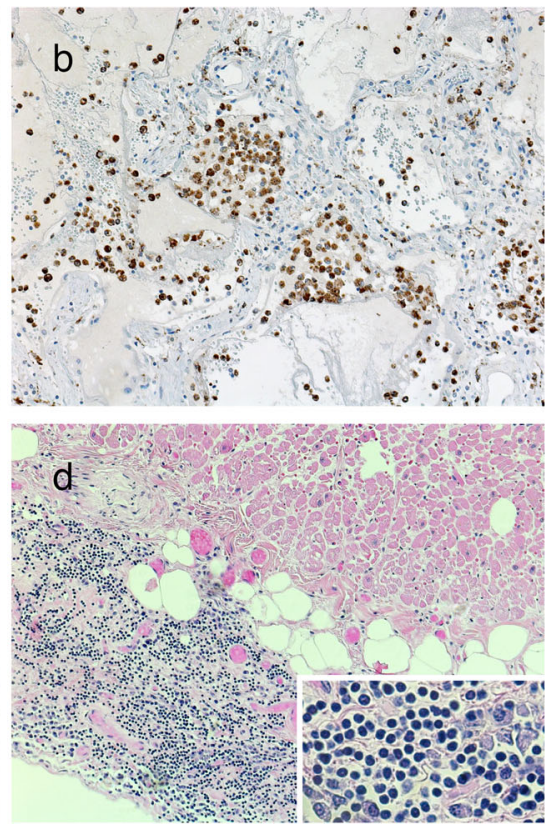

increased mitotic figures (arrow). d Low power demonstrating patchy nonspecific pericardial infiltration with aggregates of inflammatory cells (inset: high power demonstrating the predominance of lymphocytes mixed with plasma cells without neutrophils or granulomas)

However, there were no obvious signs of proliferative phase of acute lung injury or long-term processes such as squamous metaplasia $[8,9]$. The morphological features of COVID-19 resembled those seen in SARS and MERS coronavirus infection in which vascular congestion, hyaline membrane formation, and inflammatory cellular infiltration had been described [10-13]. The patchy distribution of marked atypical enlarged type II pneumocytes was characterised by large nuclei, amphophilic granular cytoplasm, and prominent nucleoli in the intra-alveolar spaces. The latter showed viral cytopathiclike changes, which indicates a severe ongoing reactive process in the lung parenchyma. In contrast to recently reported hyperplastic pneumocytes with suspected viral inclusion, no apparent intranuclear or intracytoplasmatic viral inclusions could be identified [8]. Unfortunately, there are actually no validated tests available in Europe for immunohistochemistry to confirm the presence of the virus in tissue specimens. To some degree, diffuse alveolar damage can be considered a final common pathway of several etiologies. Hence, a great number of viruses can cause the same pattern of injury. To rule out the coincidence of any other respiratory virus, a postmortem swab test of lung tissue was performed.

The deceased had been transferred to self-isolation 5 days before his death. The fact that he was found in a responsive condition 24 hours before death indicates a very rapid onset of severe acute lung injury consistent with the detected pattern injury with predominance of the early acute phase of diffuse 
alveolar damage in which the cause of deterioration remains unclear. Unfortunately, we could not get additional information regarding the clinical symptoms and his general condition during his time of self-isolation because the deceased lived alone. The detection of some neutrophils in the bronchial wall and in intra-alveolar spaces in the left lower lobe suggested the beginning of a secondary bacterial pneumonia: a phenomenon that has been described for other respiratory viruses and is associated with increased mortality rates [14]. Epidemiological studies about the influenza virus, for example, indicate that people infected with the influenza virus are most susceptible to secondary bacterial pneumonia between 4 and 14 days after the onset of influenza symptoms [15]. Nevertheless, in our case, the extent of neutrophils was limited and the main finding remains the acute diffuse alveolar damage. The proof of hyaline microthrombi and some fresh thrombi in pulmonary arteries suggest the existence of disseminated intravascular coagulation (DIC). The liver samples revealed a moderate amount of macro- and microvesicular steatosis and some single necrotic hepatocytes around the central veins without portal or lobular inflammation, indicating signs of circulatory failure or shock as a common finding before death. Thus, there was no conclusive evidence that SARS-CoV-2 induces liver injury. The tissue samples of the heart revealed a patchy non-specific inflammation of the pericardium including lymphocytes and plasma cells without neutrophils or granulomas, suggesting the presence of a viral pericarditis, as it has been described in other viral diseases [16]. However, no obvious histological changes, such as inflammatory infiltrates or substantial damage, could be detected in the myocardium. The lymph node samples revealed many non-caseous epithelioid cell granulomas as a nodal effacement of a previously unknown sarcoidosis without relevance to the cause of death. The post-mortem CT scan revealed advanced bilateral pulmonary ground-glass opacities and consolidations as an expression of advanced pulmonary disease as described in several other studies $[17,18]$. The ante-mortem blood test 6 days before death revealed a lymphopenia, which has been described frequently in patients with COVID-19 and could be considered a critical factor associated with disease severity and mortality as presented in our case [4].

\section{Conclusion}

Although several studies on the novel coronavirus (SARS$\mathrm{CoV}-2$ ) have described its characteristic radiographic findings and clinical features, there is currently a lack of pathologic data from autopsy. This case of a sudden death from severe infection during self-isolation revealed the presence of diffuse alveolar damage and atypical enlarged type II pneumocytes without obvious viral inclusions as major histopathologic findings in lung parenchyma. Furthermore, a superimposed bacterial pneumonia was suspected because of the detection of some neutrophilic infiltrates in the lower lobes. In addition, the evidence of lymphocytic infiltrate in the pericardium suggests the spread to heart tissue. However, no obvious inflammatory infiltrates could be detected within the myocardium samples. The results emphasize that the risk of ARDS development in SARS-CoV-2 infections include underlying disease such as diabetes mellitus and secondary infection. In conclusion, it is imperative to perform a complete autopsy and report the morphological findings of macroscopic examination and routine histopathology for a better understanding of the pathophysiological mechanism that causes lung injury in SARS-CoV-2 cases and to determine the cause of death with attention to underlying coinfections and comorbidities. Ultimately, it is crucial for forensic practice to know the main morphological features in Covid-19-related deaths because forensic examiners often lack detailed information about the previous clinical and medical conditions at the time of the autopsy.

Funding information This study was funded by the Institute of Legal Medicine, Cantonal Hospital St.Gallen, Switzerland

\section{Compliance with ethical standards}

Conflict of interest The authors declare that they have no conflict of interest.

\section{References}

1. Coronavirus disease 2019 (COVID-19) Situation Report -79. WHO Website. https:/www.who.int/docs/default-source/ coronaviruse/situation-reports/20200408-sitrep-79-covid-19.pdf. Accessed 08 Apr 2020

2. He F, Deng Y, Li W (2020) Coronavirus disease 2019: what we know? J Med Virol 14:1-7. https://doi.org/10.1002/jmv.25766

3. Zumla A, Chan JF, El A, Hui DS, Yuen KY (2016) Coronaviruses - drug discovery and therapeutic options. Nat Rev Drug Discov 15: 327-347. https://doi.org/10.1038/nrd.2015.37

4. Rodriquez-Morales AJ, Cardona-Ospina JA, Gutiérrez-Ocampo E et al (2020) Clinical, laboratory and imaging features of COVID19: a systematic review and meta-analysis. Travel Med Infect Dis 13:101623. https://doi.org/10.1016/j.tmaid.2020.101623

5. Wu C, Chen X, Cai Y et al (2020) Risk factors associated with acute respiratory distress syndrome and death in patients with coronavirus disease 2019 pneumonia in Wuhan, China. JAMA Intern Med 13. https://doi.org/10.1001/jamainternmed.2020.0994

6. Molina DK, DiMaio VJ (2012) Normal organ weights in men: part II-the brain, lungs, liver, spleen, and kidneys. Am J Forensic Med Pathol 33:368-372. https://doi.org/10.1097/PAF. 0b013e31823d29ad

7. Xu Z, Shi L, Wang Y, Zhang J, Huang L, Zhang C, Liu S, Zhao P, Liu H, Zhu L, Tai Y, Bai C, Gao T, Song J, Xia P, Dong J, Zhao J, Wang FS (2020) Pathological findings of COVID-19 associated with acute respiratory distress syndrome. Lancet Respir Med 8: 420-422. https://doi.org/10.1016/S2213-2600(20)30076-X

8. Tian S, Hu W, Niu L, Liu H, Xu H, Xiao SY (2020) Pulmonary pathology of early-phase 2019 novel coronavirus (COVID-19) pneumonia in two patients with lung cancer. J Thorac Oncol 28: 700-704. https://doi.org/10.1016/j.jtho.2020.02.010 
9. Tian S, Xiong Y, Liu H, Niu L, Guo J, Liao M, Xiao SY (2020) Pathological study of the 2019 novel coronavirus disease (COVID19) through postmortem core biopsies. Mod Pathol 14. https://doi. org/10.1038/s41379-020-0536-x

10. Hwang DM, Chamberlain DW, Poutanen SM, Low DE, Asa SL, Butany J (2005) Pulmonary pathology of severe acute respiratory syndrome in Toronto. Mod Pathol 18:1-10. https://doi.org/10. 1038/modpathol.3800247

11. Franks TJ, Chong PY, Galvin JR et al (2003) Lung pathology of severe acute respiratory syndrome (SARS): a study of 8 autopsy cases from Singapore. Hum Pathol 34:743-748. https://doi.org/10. 1016/s0046-8177(03)00367-8

12. Nicholls JM, Poon LL, Lee KC et al (2003) Lung pathology of fatal severe acute respiratory syndrome. Lancet 361:1773-1778. https:// doi.org/10.1016/s0140-6736(03)13413-7

13. Ng DL, Al Hosani F, Keating MK et al (2016) Clinicopathologic, Immunohistochemical, and ultrastructural findings of a fatal case of middle east respiratory syndrome coronavirus infection in the United Arab Emirates, April 2014. Am J Pathol 186:652-658. https://doi.org/10.1016/j.ajpath.2015.10.024

14. Martin-Loeches I, Van Someren GF, Schultz MJ (2017) Bacterial pneumonia as an influenza complication. Curr Opin Infect Dis 30: 201-207. https://doi.org/10.1097/QCO.0000000000000347
15. Van der Sluijs KF, van der Poll T, Lutter R et al (2010) Bench-tobedside review: bacterial pneumonia with influenza - pathogenesis and clinical implications. Crit Care 14:219. https://doi.org/10.1186/ cc8893

16. Gouriet F, Levy PY, Casalta JP, Zandotti C, Collart F, Lepidi H, Cautela J, Bonnet JL, Thuny F, Habib G, Raoult D (2015) Etiology of pericarditis in a prospective cohort of 1162 cases. Am J Med 128: 784-784.e8. https://doi.org/10.1016/j.amjmed.2015.01.040

17. Pan F, Ye T, Sun P, Gui S, Liang B, Li L, Zheng D, Wang J, Hesketh RL, Yang L, Zheng C (2020) Time course of lung changes on chest CT during recovery from 2019 novel coronavirus (COVID-19) pneumonia. Radiology 13:200370-200721. https:// doi.org/10.1148/radiol.2020200370

18. Wang D, Hu B, Hu C et al (2020) Clinical characteristics of 138 hospitalized patients with 2019 novel coronavirus-infected pneumonia in Wuhan, China. JAMA 7. https://doi.org/10.1001/jama. 2020.1585

Publisher's note Springer Nature remains neutral with regard to jurisdictional claims in published maps and institutional affiliations. 